\title{
Comparative Analysis of Particle Swarm Optimization and Particle Swarm Optimization with Aging Leader and Challengers towards Benchmark Functions
}

\author{
Anu Sharma \\ CSE Department \\ GNDU, RC Jalandhar
}

\author{
Mandeep Kaur \\ CSE Department \\ GNDU, RC Jalandhar
}

\begin{abstract}
Particle swarm optimization is the populace based heuristic optimization technique motivated by swarm intelligence and aims to find the best solution in the swarm. Aging leader and challengers with Particle swarm optimization (ALC-PSO) is a PSO variant in which concept of leader and challenger is implanted ALC- PSO has been successful in preventing premature convergence problem of PSO. In this paper, we performed experimental analysis of the performance of ALCPSO and Standard PSO Algorithm on different benchmark functions and made an effort to list out the performance differences between PSO and ALC-PSO.
\end{abstract}

\section{Keywords}

Aging, leader, particle swarm optimization, convergence, population

\section{INTRODUCTION}

Particle swarm optimization is a population based stochastic optimization technique which is proposed by Kennedy and Ebehart [1] and was intended for imitating social behavior like movement of organisms in a flock of bird or fish school. It is laid on the foundations of social influence and social learning.PSO exploits a population of optimal solutions to explore the search space.PSO relies on the information exchange between the individuals called particle of population called swarm [2]. Simplicity and ease of implementation has made PSO a popular area of research .It has wide range of applications such as fuzzy networks, power control, computer graphics, distribution, sensor and communication networks etc. PSO provides best solution for the hard problems and also used to solve real valued, binary and discrete problems[3] .But Standard PSO suffers from the problem of premature convergence in which the particle gets confined to local optima while looking for the best solution. Various PSO variants were developed to overcome this problem. In this paper we have discussed the PSO variant known as ALC-PSO proposed by Wei-Neng Chen,, Jun Zhang, Ying Lin,Ni Chen,, Zhi-Hui Zhan in 2013.In ALC-PSO i.e. particle swarm optimization with aging leader and challengers is the PSO variant in which swarm is guided by the leader particle(particle capable of Providing best optimal solution)whose lifespan is adjusted according to the leading power .If its lifespan reduce to a certain value due to poor leading power, then candidate particles (particle which provide better solution than existing leader)claim for leadership in swarm. ALC-PSO has been successful in preventing premature convergence as it generates new challengers when leader particle gets trapped into local optima. It also maintains swarm diversity. [4]This paper is organized as following. Next section covers the brief description of particle swarm optimization followed by the brief overview of the PSO with Aging leader and Challengers i.e. ALC-PSO and benchmark functions selected to evaluate performance of PSO and ALC-PSO. Next section covers the parameter setting for experiment followed by results of experiment. This section is followed by the various open issues in the field of ALC-PSO .Last section concludes the paper and lays foundation for future research.

\subsection{PARTICLE SWARM OPTIMIZATION}

This heuristic population based method consists of a problem having a swarm of probable particles, and moving these particles around in the search-space as per velocity and position update rule. A population of particles randomly positioned in an n-dimensional search space is initialized in PSO. Every particle in the swarm maintains two vectors i.e. velocity vector and a position vector. During each generation, each particle adhere update rules to update its velocity and position by knowing from the particle's previous best position and the best position found by the entire swarm so far. Let $v_{i}$ and $x_{i}$ be the velocity and position vector respectively and $M$ be no of particle in the search space or swarm. The update rules in the standard PSO are defined as

$$
\begin{aligned}
& \mathrm{v}_{\mathrm{i}}{ }^{\mathrm{j}} \leftarrow \mathrm{v}_{\mathrm{i}}{ }^{\mathrm{j}}+\mathrm{c}_{1} \cdot \mathrm{r}_{1}{ }^{\mathrm{j}} \cdot\left(\text { pbest }_{\mathrm{i}}{ }_{\mathrm{j}}-\mathrm{x}_{\mathrm{i}}{ }^{\mathrm{j}}\right)+\mathrm{c}_{2} \cdot \mathrm{r}_{2}{ }^{\mathrm{j}} \cdot\left(\text { gbest }{ }_{\mathrm{i}}{ }^{\mathrm{j}}-\mathrm{x}_{\mathrm{i}}{ }^{\mathrm{j}}\right)(1) \\
& \mathrm{x}_{\mathrm{i}}^{\mathrm{j}} \leftarrow \mathrm{x}_{\mathrm{i}}{ }^{\mathrm{j}}+\mathrm{v}_{\mathrm{i}}{ }^{\mathrm{j}} \text { (2) }
\end{aligned}
$$

In eqn.1, pbest is the best position of a particle whereas gbest is the best position of the whole swarm. $c_{1}$ and $c_{2}$ are the two constants to measure relative performance of pbest and gbest. $\mathrm{r}_{1}{ }^{\mathrm{j}}$ and $\mathrm{r}_{2}{ }^{\mathrm{j}}$ are random numbers distributed in[0,1], and $\mathrm{j}(1<\mathrm{j}<\mathrm{n}) \quad$ represents the $\mathrm{j}^{\mathrm{th}}$ dimension of the search space.[1]Comparison among particles is required for finding best position in the swarm. Convergence speed and global searching ability are the two dynamics for evaluating the functioning of PSO algorithms. Original PSO algorithm exhibits fast-converging behavior as gbest updates velocities and distance. But In multimodal problems, a best position confined to a local optima may take in the entire swarm leading to premature convergence .Many PSO variants developed to improve the performance of PSO achieve the preservation of swarm diversity at the cost of slow convergence. It is difficult to avoid premature convergence without worsening the speed of convergence and the simplicity of the structure of PSO. 


\subsection{PSO WITH AGING LEADER AND CHALLENGERS ALGORITHM}

ALC-PSO algorithm is introduced for solving problem of premature convergence in PSO and maintains fast converging features of PSO. ALC-PSO differentiates itself from the original PSO in such a way that the leader of the swarm ages within a limited lifespan. The lifespan of leader is adjusted according to the leader's leading power. When the lifespan is expired, the leader is challenged and replaced by newly generated particle with better leading power. In ALC-PSO velocity update formulae, gbest is replaced by particle with the best leading power i.e. leader .The velocity and position update rules for ALC-PSO are given as follows:

$v_{i}{ }^{j} \leftarrow w \cdot v_{i}{ }^{j}+c_{1} \cdot r_{1}{ }^{j} \cdot\left(\right.$ pbest $\left._{i}{ }_{i}{ }-x_{i}{ }^{j}\right)+c_{2} \cdot r_{2}{ }^{j} \cdot\left(\right.$ Leader $\left.^{j}-x_{i}{ }^{j}\right)(3)$

$\mathrm{x}_{\mathrm{i}}^{\mathrm{j}} \leftarrow \mathrm{x}_{\mathrm{i}}^{\mathrm{j}}+\mathrm{v}_{\mathrm{i}}^{\mathrm{j}}(4)$

$\mathrm{w}$ in the equation (3) is inertia weight whose large value leads to global search and smaller value leads to local search. value of inertia weight affect convergence. In ALC-PSO, as soon as the leader traps into local optima, new challengers are generated to claim leadership of swarm and lead the swarm towards best solution. On straightforward unimodal functions, it is normally simple for the leader to enhance the nature of the swarm and consequently the leader has solid driving force. For this situation, the leader has a longer lifespan to lead the swarm and the pursuit conduct of ALC-PSO is fundamentally the same to that of the original PSO. Subsequently, the fast converging feature of the PSO can be protected. whereas on complex multimodal functions, once the leader confines to local optima, it neglects to enhance the nature of the swarm and gets matured rapidly. For this situation, new challengers rise to supplant the old leader and bring in differences. In terms of search speed, ALC -PSO is the quickest algorithm of all other PSO algorithms. ALC-PSO figures out how to get results with high precision on these multimodal functions regarding function evaluations and execution time. ALC-PSO performs better than the other enhanced PSO variations on unimodal functions.

The Steps involved in ALC-PSO is given as follows:

Step 1: Initialization: The initial positions of all particles are generated randomly within the n-dimensional search space. Velocities of particles are initialized to 0 . The best particle among the swarm is chosen to be the Leader. The age of the leader is initialized to zero and the lifespan of the leader is set to an initial value 0 .

Step 2: Velocity and Position Updating: Every particle follows the velocity update rule and the position update rule to adjust its velocity and position.

Step 3: Updating leader: For particle $\mathrm{i}(\mathrm{i}=1,2, \ldots, \mathrm{M})$, if the newly generated position is better than Leader then the new generated particle becomes the new Leader of the particular population. If best position found in this iteration is better than the leader then leader is updated. In this way the Leader represents the best solution generated by particles during the leader's lifetime.

Step 4: Lifespan Control: After the positions of all particles are updated, the leading power of the Leader to improve the entire swarm is evaluated. The lifespan $b$ is adjusted by a lifespan controller. When the leader has strong leading power the controller increases its lifespan .On the other hand if leading power of leader is poor, then controller decreases the lifespan of leader.
Step 5: Generating a Challenger: A newly generated particle with better position challenges the Leader whose lifespan is finished.

Step 6: Evaluating the Challenger: The leading power of the newly generated challenger is evaluated and compared with the leading power of existing leader. If the challenger has enough leading power, it replaces the old Leader and becomes the new Leader. Otherwise, the old Leader continues to be the leader of the swarm.

Step 7: Terminal Condition Check: Check whether the number of function evaluations exceed the maximum evaluations, if yes then terminate the algorithm else go to step 2 for another round of iteration.[4][5]

\subsection{BENCHMARK FUNCTIONS}

In this we are going to discuss the various Benchmark functions on which we will test the performance of PSO and ALC-PSO. Benchmark functions are presented with the aim of giving an idea about the different situations that optimization algorithms have to face when coping with these kinds of problems.

a) Ackley test function: It has several local optima that, for the search range [-32, 32], look more like noise, although they are located at regular intervals. The Ackley function only has one global optimum located at the point 0. [9]Function definition is mentioned below :

$$
f(x)=20+e^{-20} \exp \left(-20 \sqrt{1 / n} \sum_{i=1}^{n} x_{i}^{2}\right.
$$

b) Sphere test function : It is one of the most simple test functions available in the specialized literature. This test function can be scaled up to any number of variables. It belongs to a family of functions called quadratic functions and only has one optimum in the point $0 .[10]$ The search range commonly used for the Sphere function is $[-100,100]$ for each decision variable. Function definition for spherical function is given as follows

$$
f(x)=\sum_{i=1}^{n} x_{i}^{2}
$$

c) Schwefel test function : It is a quadratic function. It also has only one optimum and its search range is the same as that of the Sphere function (i.e., $[-100,100]$ for each variable).[10] Schwefel test function is given as

$$
f(x)=-\sum_{i=1}^{n} x_{i} \sin \left(\sqrt{\left|x_{i \mid}\right|}\right)
$$

d) Cigar Test Function :This is a multimodal objective function whose search range is $[-100,100]$ with the function definition mentioned below

$$
f(x)=x_{1}^{2}+10^{6} \sum_{i=2}^{n} x_{i}^{2}
$$

e) SumCan Test Function :This is a Objective Function whose search range is $[-0.16,0.16]$ and global optimum at $-10^{5}$. Function definition of sum can benchmark function[9] is 


$$
f(x)=-\left\{10^{5}+\sum_{i=1}^{n}\left|y_{i}\right|\right\}^{-1}
$$

\section{EXPERIMENTAL SETTINGS AND \\ RESULTS}

Empirical analysis needs to be performed to investigate the behavior of an algorithm. So, In this section we evaluated the performance of the PSO and ALC-PSO by examining the gbest values obtained after performing experiments. Evaluation of the gbest value of particles in swarm is performed at 5 different benchmark functions. At the end, the gbest values obtained for PSO and ALC-PSO algorithms are compared.

Parameters Settings for the experiment is mentioned below:

- $\quad$ Initial Population $=10$

- Cognitive constant $\mathrm{c} 1=2$

- $\quad$ social constant $\mathrm{c} 2=2$

- Inertia weight, $\mathrm{w}=0.9$

- $\quad$ Maximum evaluations $=20$

The table given below clearly shows that the ALC-PSO provide better solutions than the standard PSO. gbest values are better in ALC-PSO as values are more close to the optimum solution than values provided by standard PSO. gbest depicts the best position obtained in the whole swarm. Table clearly presents that In most of the test problems, PSO with Aging leader and challengers provides better solution. Significant performance improvement has been seen on Sumcan test function whereas ALC-PSO did not perfomed better than PSO on Ackley function. The above results were obtained after implementation of PSO and ALC-PSO algorithms on MATLAB(R2010a).

Table 1.Comparison of PSO and ALC-PSO

\begin{tabular}{|c|c|c|}
\hline Algorithm & PSO & ALC-PSO \\
\hline $\begin{array}{c}\text { Ackley } \\
\text { Function }\end{array}$ & 0.0239 & 0.0532 \\
\hline $\begin{array}{c}\text { Sphere } \\
\text { Function }\end{array}$ & 0.2849 & 0.0814 \\
\hline $\begin{array}{c}\text { Schwefel } \\
\text { Function }\end{array}$ & 1.0559 & 0.0556 \\
\hline $\begin{array}{c}\text { Cigar } \\
\text { Function }\end{array}$ & 0.4935 & 0.0000 \\
\hline $\begin{array}{c}\text { Sumcan } \\
\text { Function }\end{array}$ & 74.3639 & 0.0289 \\
\hline
\end{tabular}

\subsection{GRAPHS GENERATIONS DEPICTING COMPARISON OF PSO} AND ALC-PSO

In this Section, we presented the graphs obtained on running PSO and ALC-PSO algorithms on MATLAB. These graphs represent the error generated at each iteration on different benchmark functions is depicted below for PSO as well as ALC-PSO code. Error is defined as the deviation of the obtained results from the optimum value. lesser the deviation ,better are the results. From the graphs, It is clearly evident that the error rate is lesser for ALC-PSO for most of the benchmark functions as compared to the PSO algorithm. From results, it is evident that ALC-PSO has been successful in providing best optimal results in comparison to PSO and also delays convergence to a greater extent. ALC-PSO converge to the optimal solutions at later stages whereas PSO converges to the Optimal solution at early stages. Problem of premature convergence that occurs in PSO has been overcame by introduction of aging concept in PSO i.e. ALC-PSO.

From graphs, it is evident that ALC-PSO performed better than PSO on all the test functions except Ackley test function. For Cigar and Schwefel Test functions, error rate of ALCPSO increased with the increasing iterations. For sphere and Sumcan test function, error rate started decreasing at later iterations. Graphical representation of comparison between PSO and ALC-PSO on different benchmarks functions is shown below. 


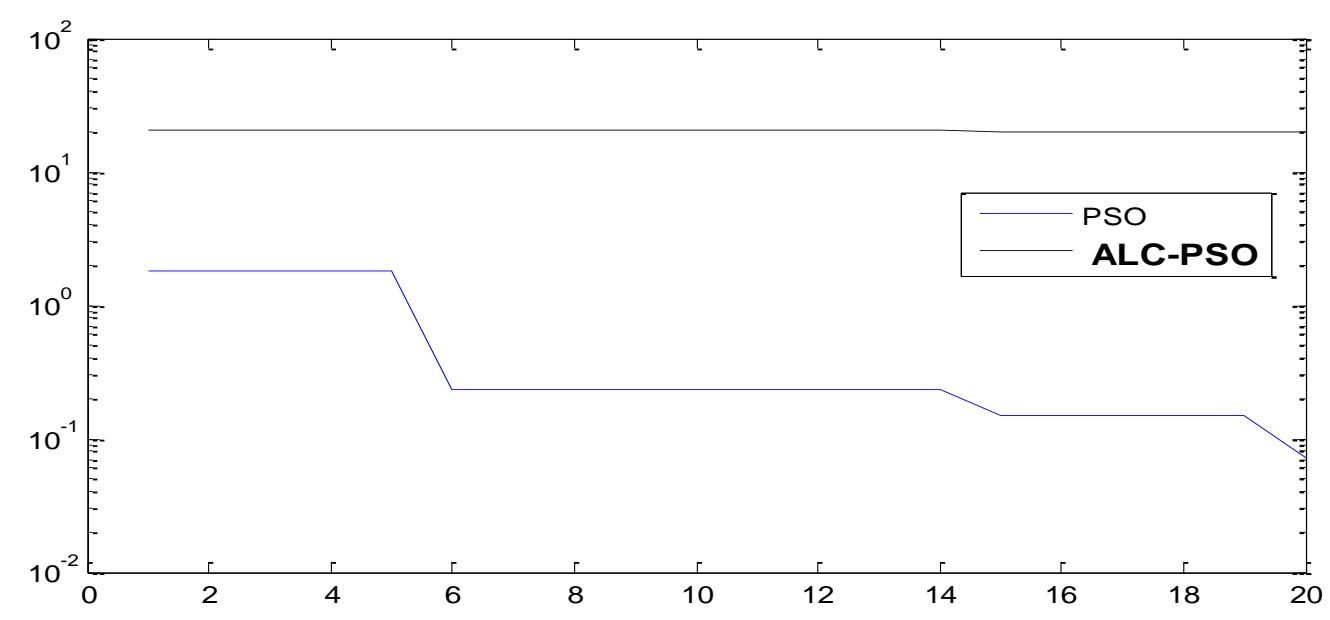

Fig 1.Comparison of PSO and ALC-PSO on Ackley Test Function

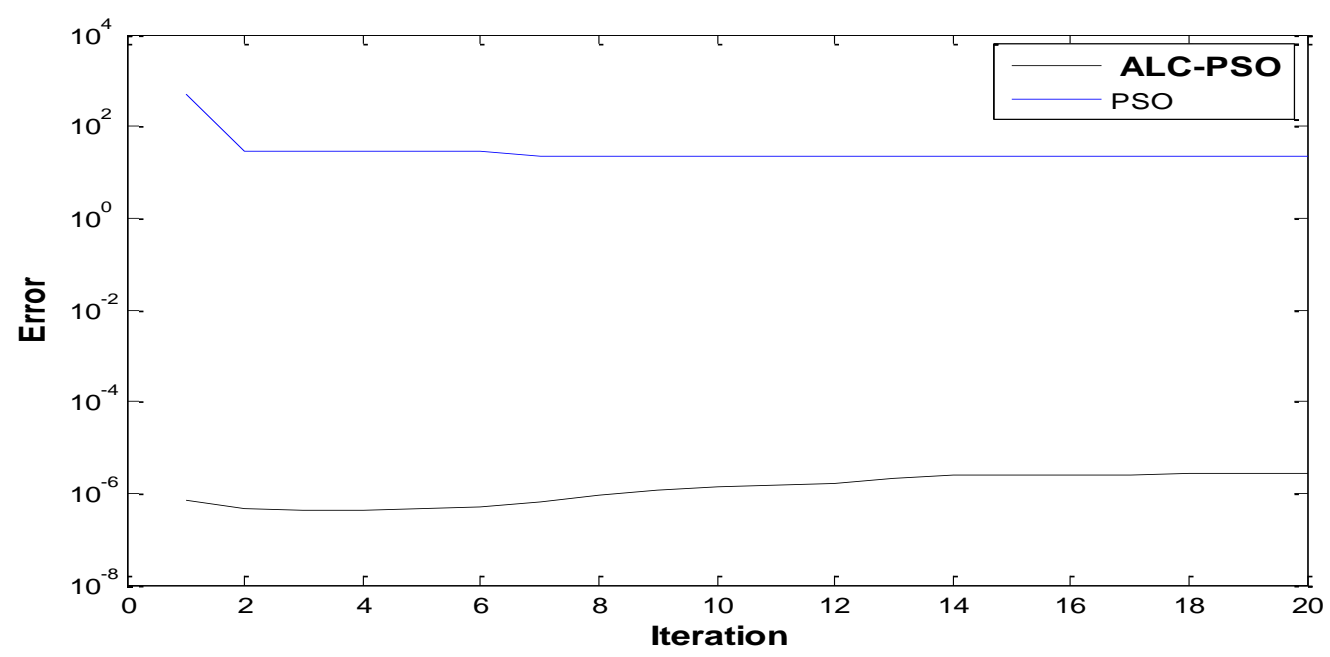

Fig 2 . Comparison of PSO and ALC-PSO on Cigar Test Function

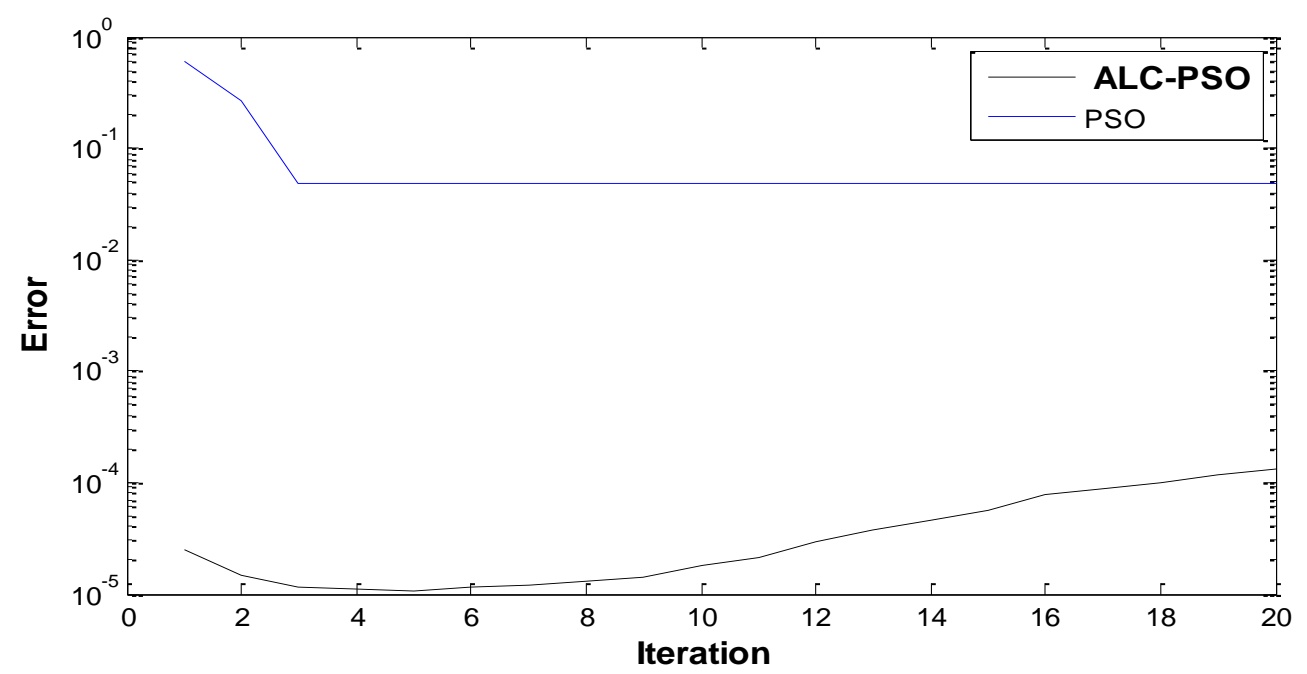

Fig 3.Comparison of PSO and ALC-PSO on Schwefel Function 


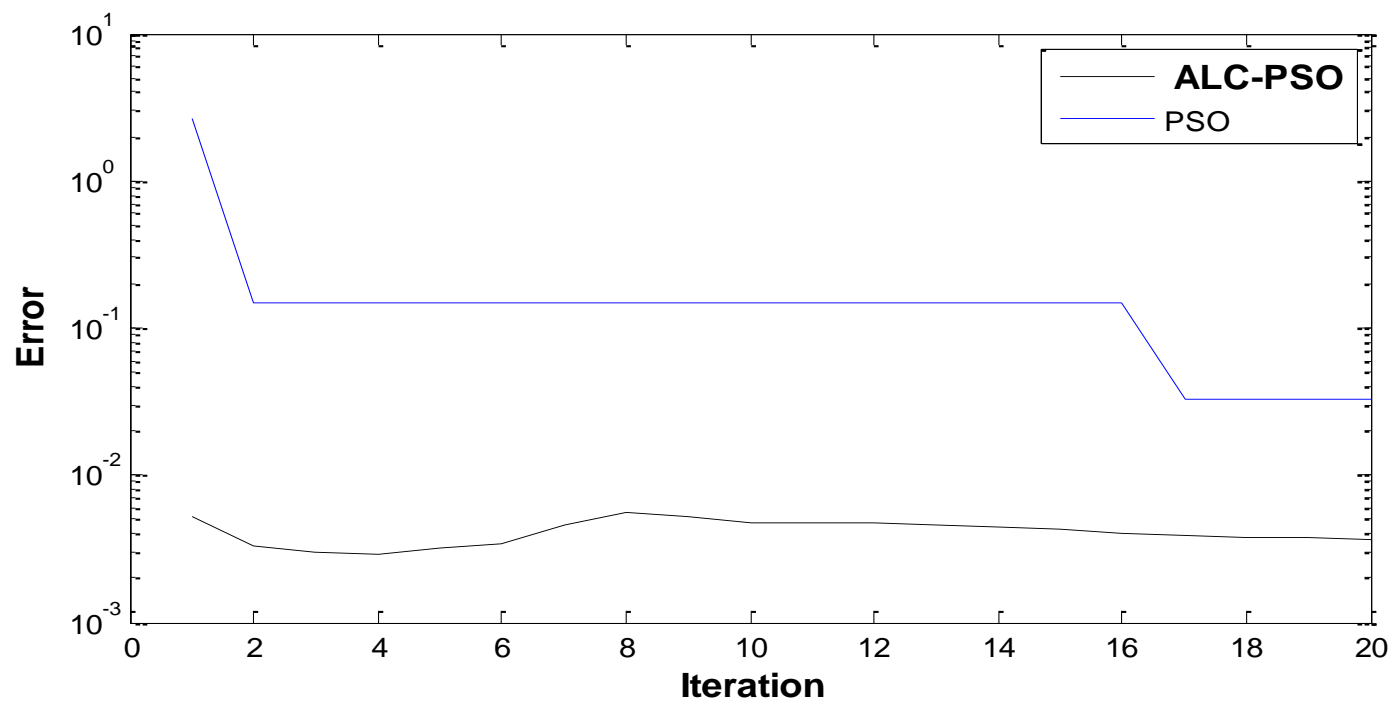

Fig 4 .Comparison of PSO and ALC-PSO on Sphere Test Function

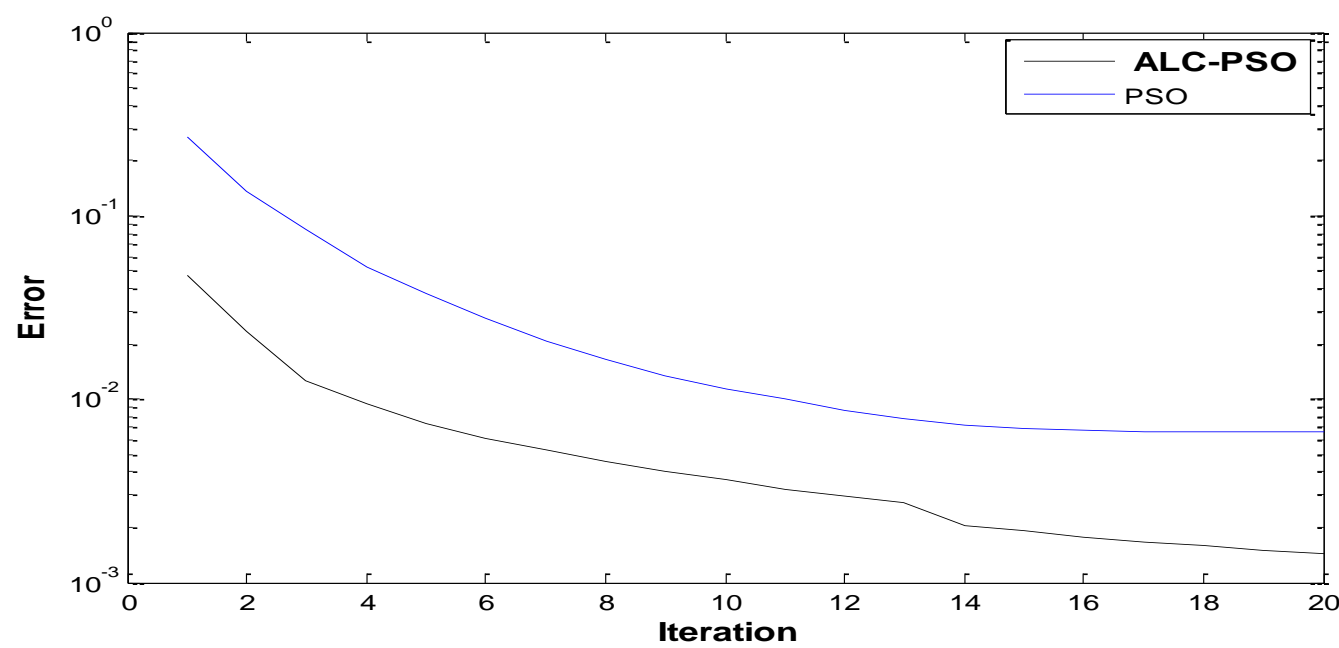

Fig 5.Comparison of PSO and ALC-PSO on Sumcan Test Function

\section{OPEN ISSUES}

Considerable amount of work has been done in direction of improving performance of PSO by introduce numerous variations into it. ALC-PSO is one of those variants which significantly improved the performance of PSO But there are certain issues which has not been resolved yet are listed below

- $\quad$ Side constraints may get infringed in ALC-PSO when a particle shifts to a new position.

- Time for searching global optimal solutions has not been reduced.

- $\quad$ Aging concept would be difficult to be implemented in complex problems as it will incur complexity overhead.

- $\quad$ Search time for finding new leader needs to reduced.

- High computational time.

\section{CONCLUSION AND FUTURE SCOPE}

PSO is stochastic mechanism used to discover a best arrangement much quicker than the other improvement techniques. Straightforward idea and simple usage of PSO has made it mainstream among researchers. Concept of aging is introduced in PSO to conquer the issue of untimely convergence in standard PSO. This aging based PSO variant is known as ALC-PSO has gotten to be main stream among scientists of distinctive fields as it gives ideal execution over standard PSO. ALC-PSO can be used in various application such as image enhancement, sensor networks, combinatorial problems etc. in future to enhance the performance provided by the PSO in those application. ALC-PSO can be tested on complex optimizations problems. Effect of parameter variation can be investigated on ALC-PSO. Concept of aging can be incorporated in other evolutionary techniques such as $\mathrm{ACO}, \mathrm{DE}$ etc.

\section{ACKNOWLEDGEMENT}

I would like to thank my teachers, parents and my friends for all their support in this paper.

\section{REFERENCES}

[1] J. Kennedy and R. C. Eberhart, "Particle swarm optimization," in Proc. IEEE Int. Conf. Neura Netw., Nov.-Dec. 1995, pp. 1942-1948.

[2] Konstantinos E. Parsopoulos and Michael N. Vrahati ,“ Particle Swarm Optimization Method for Constrained 
Optimization Problems", In Proceedings of the EuroInternational Symposium on Computational Intelligence 2002 .

[3] Russell C. Eberhart, Yuhui ShiGuest Editorial Special Issue on Particle Swarm Optimization" IEEE TRANSACTIONS ON EVOLUTIONARY COMPUTATION, VOL. 8, NO. 3, JUNE 2004.

[4] Y. Shi and R. C. Eberhart, -A modified particle swarm optimizer,\| in Proc. IEEE Congr. Evol. Comput., May 1998, pp. 69-73.

[5] Shailendra S. Aote ,A Brief Review on Particle Swarm Optimization: Limitations \& Future Directions", International Journal of Computer Science Engineering (IJCSE) .

[6] Riccardo Poli, "Analysis of the Publications on the Applications of Particle Swarm Optimisation" Journal of Artificial Evolution and Applications Volume 2008, Article ID 685175, 10 pages doi:10.1155/2008/685175.

[7] Wei-Neng Chen,, Jun Zhang, Ying Lin,Ni Chen,, ZhiHui Zhan,, Henry Shu-Hung Chung, Yun Li, and YuHui Shi," Particle Swarm Optimization with an Aging
Leader and Challengers" Ieee transactions on evolutionary computation, vol. 17, no. 2, april 2013\

[8] S.Vijayalakshmi, D.Sudha, S.Mercy Sigamani, K.Kalpana Devi "Particle Swarm Optimization with Aging Leader and Challenges for Multiswarm Optimization ",International Journal of Advanced Research in Computer Engineering \& Technology (IJARCET) Volume 3 Issue 3, March 2014.

[9] Millie Pant, Radha Thangaraj, and Ajith Abraham," Particle Swarm Optimization: Performance Tuning and Empirical Analysis", Foundations of Comput. Intel. Vol. 3, SCI 203, pp. 101-128. springerlink.com (c) SpringerVerlag Berlin Heidelberg 2009.

[10] Bahareh Nakisa, Mohd Zakree Ahmad Nazri, Mohammad Naim Rastgoo and Salwani Abdullah "a survey: particle swarm optimization based algorithms to solve premature convergence problem", Journal of Computer Science 10 (9): 1758-1765, 2014 ISSN: 15493636 @ 2014 Science Publications. 